\title{
Data Hiding Techniques: A Review
}

\author{
Shweta Patil \\ Student, Electronics \\ Amrutvahini college of engineering, Sangamner \\ Maharashtra, India
}

\author{
S. S. Katariya \\ Assistant Professor \\ Amrutvahini college of engineering, Sangamner \\ Maharashtra, India
}

\begin{abstract}
In recent years, due to explosive growth in the use of internet the security of sensitive data becomes one of the prime concern. For this various data hiding techniques are in used. Now a days reversible data hiding in encrypted images is in used due to its excellent property which is original cover can be recovered with no loss after extraction of the embedded data. Also it protect the original data. Previously all the methods use vacate room after the encryption (VRAE) method but the problem with that is sometimes errors are occurred at the time of recovery of original image. So we use reversible data hiding by reserving room before the encryption. Also we survey the reversible data hiding techniques with different algorithms used
\end{abstract}

\section{Keywords}

Data hiding, cover media, RDH, MVD, encryption

\section{INTRODUCTION}

Data hiding is defined as the process of embedding the message signal into the host or cover of image to get the composite signal. Following are the 3 main requirement of data hiding system,

\section{Perceptual Transparency \\ 2. Robustness \\ 3. Capacity}

Mainly data hiding techniques are classified into two techniques:

\section{Reversible data hiding technique:}

In this technique the message signal as well as the original cover can be with no loss recovered simultaneously.

\section{Irreversible data hiding technique:}

In this technique the message signal can be recovered with no loss but the original cover can be lost.

So in general reversible data hiding techniques can be used now a days.

Method of reversible data hiding are reserving room before encryption and vacating room after encryption.

1) Vacating Room Before the Encryption:

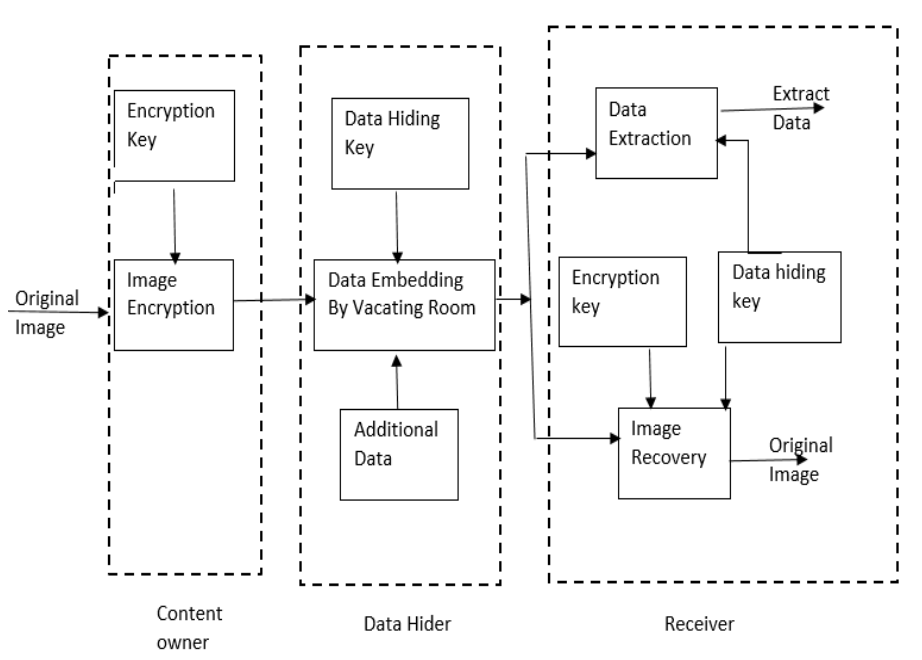

Fig: Vacating Room after Encryption (VRAE)

In this method first encrypt the original image using the cipher with the encryption key. Next to this it is given to the data hider to hide some auxiliary data in it by with no loss vacating the room required for data hiding key. At receiver the content owner or an authorized third party can be extract the embedded data with the help of data hiding key and also recover the original image according to the encryption key. This method compresses the encrypted LSBs of image to vacate the room for additional data by finding syndromes of a parity check matrix.

3. Reserving room before the encryption:

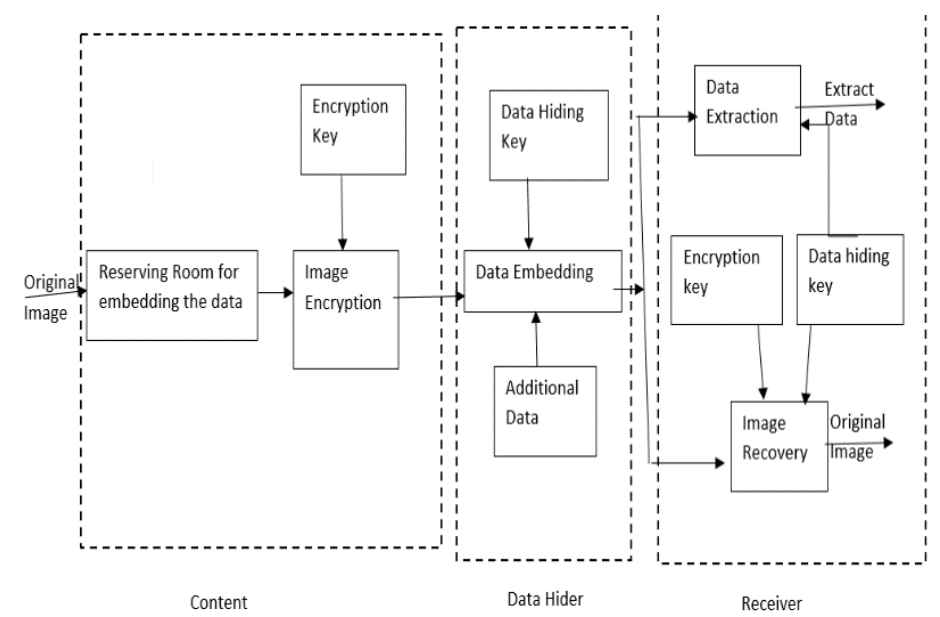

Fig: Reserving Room before Encryption (RRBE) 
In this the enough space is reserved before the encryption of the original process. After this the encryption and then data hiding as we done in above process. There are four main steps in this process: 1.Encryption of image 2.Data hiding 3. Data extraction 4 . Image recovery

\section{LITERATURE REVIEW}

Up till now more work or research has been done in reversible data hiding techniques. Lots of efficient techniques are proposed till. The work done in reversible data hiding techniques are as follows:

We know it is very difficult to vacate the room losslessly from encrypted images. So by considering this in [1] Kede Ma, Weiming Zhang, Xianfeng Zhao, Nenghai Yu and Fenghua Li has proposed a technique for embedding the data in reversible manner using reserve room before encryption.

In [2] Jui Tian has introduced another technique. They introduced a difference expansion technique. In this the space for the data embedding is discovers by exploring the redundancy in the given image. This technique is the best in among all the technique in literature survey because the holding capacity of secret data and the visual quality of embedded images. Also the computational complexity of this is low as compared to other.

In [3] Wen-Chung Kuo, Po-Yu Lai, Lih-ChyauWuu has proposed a adaptive reversible data hiding method. A new scheme based on histogram and slope method enhancing the data hiding capacity and also the efficiency increases and maintains the high quality of image.

In [4] Kuo-Ming, Wen-Kai Su, Ting-Wen Chen, Li-Ming Chen has proposes the method which is the combination of data hiding, half-toning and vector quantization technique. In this the embedding of gray scale image in other image is done. This scheme is work as follows:

a) Image compression from gray scale to half tone using half-toning process.

b) Computation of difference between original image and the image on which we perform operations.

c) Then VQ compression compress the difference obtain in above and embed it with secret data.

José .R; Abraham .G, in [6] proposed the method in which a reversibly data hiding in encrypted gray scale image is done in separable manner. Encryption of the image is done by content owner using encryption key by permuting the pixels. Data hiding is done by using data hiding key using histogram modification method.

In [7] Naor introduced the visual cryptography. The proposed scheme is the combination of reversible data hiding and colour visual cryptography.

Malik, Anjali Sardana, Jaya in [8] has proposed another different approach for visual cryptography which consist of three steps:

1.Sieving 2. Division 3. Shuffling to generate random shares. The advantage of this is that minimal computation requirement to generate the binary secret image without loss of quality of image.
In [9] InKoo Kang, Gonzalo R. Arce , Heung-Kyu Lee introduces a new method which produces the colorful meaningful shares based on pixel synchronization and halftoning of error diffusion.

In [10] Wei Qiao, Hongdong Yin, Huaqing Liang has proposed a novel method of visual cryptography based on halftone for visual cryptography. There are three main steps involve and they are as follows: first chromatic image is dissolved into three monochromatic images having tone cyan, magenta and yellow. Secondly transformation of these three images into binary image is done using halftone technique. Next to that to get sharing images the traditional binary sharing scheme is used.

Yi-Hui Chen, Ci-Wei lan and Chiaio-Chih Huang in [11] have proposed the another different approach for visual cryptography is authentication mechanism. In this two procedures are there :

a) Encryption procedure

b) Decryption procedure

Decryption of secret image and authenticated image is obtain by difference expansion.

The summery of literature survey is as shown in table:

\begin{tabular}{|c|c|c|}
\hline 'Paper & Algorithm Used & Result (PSNR Value) \\
\hline $\begin{array}{l}\text { A Reversible Data } \\
\text { Hiding Method for } \\
\text { Encrypted Images }\end{array}$ & AES algorithm & $66.13 \mathrm{~dB}$ \\
\hline $\begin{array}{l}\text { Information Hiding } \\
\text { Based on Block } \\
\text { Match Coding for } \\
\text { Vector Quantization- } \\
\text { Compressed Images }\end{array}$ & BMC algorithm & $29.1 \mathrm{~dB}$ \\
\hline $\begin{array}{l}\text { Reversible Data } \\
\text { Hiding using Visual } \\
\text { Cryptography }\end{array}$ & $\begin{array}{l}\text { Reserving room before } \\
\text { encryption }\end{array}$ & $28 \mathrm{db}$ \\
\hline $\begin{array}{l}\text { Robust } \\
\text { Watermarking of } \\
\text { Compressed } \\
\text { and Encrypted } \\
\text { JPEG2000 Images }\end{array}$ & $\begin{array}{l}\text { Spread Spectrum (SS), } \\
\text { Scalar Costa Scheme } \\
\text { Quantization } \\
\text { IndexMedulation (SCS- } \\
\text { QIM), } \\
\text { and Rational Dither } \\
\text { Modulation (RDM). }\end{array}$ & $21 \mathrm{db}$ \\
\hline $\begin{array}{l}\text { Content based } \\
\text { watermarking of } \\
\text { images }\end{array}$ & $\begin{array}{l}\text { Edge separation and } \\
\text { image segmentation }\end{array}$ & $20 \mathrm{db}$ \\
\hline $\begin{array}{l}\text { Separable } \\
\text { Reversible Data } \\
\text { Hiding in Image } \\
\text { Using } \\
\text { Advanced } \\
\text { Encryption Standard } \\
\text { with Fake Data } \\
\text { Generation }\end{array}$ & AES algorithm & $\begin{array}{l}\text { Same as } 1^{\text {st }} \text { one only } \\
\text { difference is in this we } \\
\text { differentit fake data also }\end{array}$ \\
\hline
\end{tabular}




\section{CONCLUSION}

Reversible data hiding in encrypted images is a new topic drawing attention because of the privacy-preserving requirements from cloud data management.

Previous methods implement RDH in encrypted images by vacating room after the Encryption ,as opposed to which we proposed by reserving room before encryption. Also in this we survey different techniques for data hiding along with different algorithms..

\section{REFERENCES}

[1] Kede Ma. Weiming Zhang, Xianfeng Zhao, Nenghai $\mathrm{Yu}$, Fenghua Li, "Reversible Data Hiding in Images by Reserving Room Before Encryption", IEEE Trans on Information Forensics and security, Vol. 8, No. 3, March 2013

[2] Jun Tian, "Reversible Data Embedding Using a difference Expansion", IEEE Transaction, Vol.13, No. 8, Aug 2003

[3] Wen Chung Kuo, Po Yu Lai, Lih Chyau Wuu, "Adaptive Reversible Data Hiding Based on Histogram", 10th International Conference on Intelligent Systems Design and Application, l' IEEE 2010 (2002) The IEEE website. [Online]. Available: http://www.ieee.org/

[4] Kuo-Ming Hung, Wen-Kai Su, Ting-Wen Chen, Li-Ming Chen, "Reversible Data Hiding Base on VQ and Halftoning Technique", International Conference on Microelectronics, Communication and Renewable Energy (ICMiCR-2013).

[5] Yun Q. Shi, "Reversible Data Hiding", I.J. Cox et al.: IWDW 2004, LNCS 3304, pp. 1-12 2005 l' SpringerVerlag Berlin Heidelberg 2005 "PDCA12-70 data sheet," Opto Speed SA, Mezzovico, Switzerland
[6] Jose, R.; Abraham, G, "A separable reversible data hiding in encrypted im age with improved performance", Emerging Research Areas and 2013 International Conference on Microelectronics, Communications and Renewable Energy( AICERA/ICMiCR), 2013 Annual International Conference l'IEEE 2013.

[7] Moni Naor, Adi Shamir," Visual Cryptography", in Proc EUROCRYPT'94, Berlin, Germany, 1995, vol. 950, pp. 1-12,Springer-Verlag, LNCS

[8] Siddharth Malik, Anjali Sardana, Jaya, "A Keyless Approach to Image Encryption", 2012 international conference on Communication systems and Network Technologies l'2012 IEEE

[9] InKoo Kang, Gonzalo R. Arce , Heung-Kyu Lee, " Color Extended visual cryptography using error diffusion", ICASSP 2009 1' IEEE 2009

[10] Wei Qiao, Hongdong Huaqing Liang, "A kind of Visual Cryptography Scheme For color Images based on halftone technique", International Conference on Measuring Technology and Mechatronics automation 1' 2009 IEEE

[11] Yi-Hui Chen, Ci-Wei lan and Chiao Chih Huang, " A verifiable Visual Cryptography Scheme", Fifth International Conference and Evolutionary Computing l' IEEE 2011

[12] Dawen Xu, Rangding Wang, and Yun Q. Shi, Fellow, IEEE "Data Hiding in Encrypted H.264/AVC Video Streams by Codeword Substitution" IEEE Transaction On Information Forensics and Security, Vol. 9, NO. 4 April 2014 applied to machine vibration analysis," in Proc. ASCI, 1999, pp. 398-405. 